\title{
Outcomes and safety of bronchial artery embolization in control of massive hemoptysis Gehan H. AboEl-Magd ${ }^{a, c}$, Ahmad H. Abouissa ${ }^{\mathrm{b}, \mathrm{d}}$, Mohamed M. Harraz ${ }^{\mathrm{b}}$
}

\author{
Background The most frequent causes of massive \\ hemoptysis are bronchiectasis, myecetoma, tuberculosis, \\ bronchial carcinoma, and cryptogenic hemoptysis.
}

Objective This study aimed to investigate the outcomes, safety, and complications of bronchial artery embolization (BAE) in the management of massive hemoptysis.

Patients and methods This study included 32 patients who presented with massive hemoptysis who were indicated for BAE. The following data were obtained from each patient: detailed history, clinical examination, chest radiograph, computed tomography pulmonary angiography, fiberoptic bronchoscopy, and BAE.

Results Fifteen patients underwent BAE as an urgent procedure for control of massive hemoptysis and 17 patients underwent BAE as an elective procedure. The complications of BAE were fever in four patients $(12.5 \%)$, back pain in three $(9.38 \%)$, failure of BAE in two $(6.25 \%)$, recurrence of hemoptysis in one $(3.13 \%)$, and no mortality. BAE was successful in 30 out of $32(93.75 \%)$ cases. Failure of catheterization was encountered in one $(3.13 \%)$ case because of dissection during negotiations; therefore, no catheterization was performed. The second $(3.13 \%)$ case showed extensive bronchial-pulmonary shunt with severe lung destruction, so that the procedure was deemed futile.

\section{Introduction}

Massive hemoptysis is defined as the amount of expectorated blood that endangers the airway patency, causing hemodynamic instability [1,2]. There is no definitive volume of expectorated blood for the diagnosis of massive hemoptysis; however, the amounts of hemoptysis ranging from 200 to $1000 \mathrm{ml}$ over $24 \mathrm{~h}$ are considered to be indicative of massive hemoptysis [2].

Massive hemoptysis originates from the bronchial high-pressure circulation in $90 \%$ of the cases. In around 5\%, bleeding arises either from the aorta secondary to the aortobronchial fistula, ruptured aneurysms, or systemic nonbronchial circulation. In the remaining $5 \%$, pulmonary vessels may be the source of massive hemoptysis $[3,4]$.

The most frequent causes of massive hemoptysis are bronchiectasis, myecetoma, tuberculosis (TB), bronchial carcinoma, and cryptogenic hemoptysis; however, TB is considered the most common cause, especially in countries with a high prevalence of TB [5]. Hemoptysis can occur as a sequelae of destruction of the lung vasculature and parenchyma because of pulmonary TB [6].
After the procedure, immediate control of hemoptysis was achieved in 29 (90.62\%) patients.

Conclusion BAE can be used safely and effectively for control of massive hemoptysis; it can be used as an alternative nonsurgical option along with medical treatment or as a bridge to stabilize the patient until definitive surgical management can be performed. BAE can be considered an alternative to surgery if the patient is not fit for surgical intervention.

Egypt J Bronchol 2019 13:666-671

(C) 2020 Egyptian Journal of Bronchology

Egyptian Journal of Bronchology 2019 13:666-671

Keywords: bronchial artery, bronchoscopy, embolization, hemoptysis

aDepartment of Chest Diseases, Faculty of Medicine, Tanta University, Tanta, ${ }^{b}$ Radiology Department, Faculty of Medicine, Mansoura University, Mansoura, Egypt, 'Chest Department, ${ }^{\circ}$ Interventional Radiology Department, Al-Noor Specialist Hospital, Makkah, Saudi Arabia

Correspondence to Gehan H. AboEl-Magd, MD, Assistant Professor of Chest Diseases, Tanta University, Tanta, 31111, Egypt. Tel: 01006413962; e-mail: gehan19@yahoo.com

Received: 10 June 2019 Accepted: 10 September 2019 Published: 21 January 2020

The lung has dual circulation with bronchial and pulmonary; the first one supplies the tracheobronchial tree down to the level of the terminal bronchiolesand the pulmonary circulation is involved in gas exchange of oxygen and carbon dioxide [7].

Control of massive hemoptysis by conservative methods leads to a high rate of mortality varying from 50 to $100 \%$ [8]; the common cause of death is asphyxiation [9].

If the cause of hemoptysis is not known, especially in patients with suspected malignancy, bronchoscopy should be performed. Computed tomography (CT) chest is also recommended before bronchoscopy. Both procedures are considered complementary, increasing the diagnostic value for the source of bleeding [10].

This is an open access journal, and articles are distributed under the terms of the Creative Commons Attribution-NonCommercial-ShareAlike 4.0 License, which allows others to remix, tweak, and build upon the work non-commercially, as long as appropriate credit is given and the new creations are licensed under the identical terms. 
CT chest is useful for the diagnosis of cancer or bronchiectasis, whereas bronchoscopy has a better diagnostic yield in the detection of mucosal abnormalities [11]. In addition, bronchoscopy can provide specimens of BAL and biopsies for culture, cytology, and histopathology [12].

\section{Objective}

This study aimed to investigate the outcomes, safety, and complications of bronchial artery embolization (BAE) in the management of massive hemoptysis.

\section{Patients and methods}

This study was carried out during the period from December 2014 till February 2019 in the radiology Department in Al-Noor Specialist Hospital, Makkah, Saudi Arabia. It included 32 patients who presented with massive hemoptysis in whom the amount of bleeding was 200-1000 $\mathrm{ml}$ over $24 \mathrm{~h}$ who were indicated for BAE as a first line of management of massive hemoptysis, failure or contraindications of bronchoscopy or bridge to surgery to stabilize the patient. The study was approved by the local institute ethics committee.

The following data were obtained from each patient: detailed history, clinical examination, chest radiograph, CT pulmonary angiography, and updated coagulation profiles that were reviewed before fiberoptic bronchoscopy and BAE. Bronchoscopy guides the radiologist to the proper segment for embolization and therefore reduces the duration of BAE. CT scan provides an anatomy of the bronchial arteries and is considered a roadmap for BAE.

All patients were scheduled for regular follow-up at least for 1 year following BAE; recurrence was considered when bleeding occurred again within this year.

Inclusion criteria were as follows: patients with pulmonary causes of hemoptysis proven by $\mathrm{CT}$ pulmonary angiography, for example, TB were eligible for this study. Exclusion criteria were as follows: patients presented with nonpulmonary causes of massive hemoptysis, for example, thrombocytopenia, platelet disorders were excluded from the study.

Benefits, risks, and alternatives were discussed with the patient or guardians before obtaining an informed consent from all patients. Patient consent declaration was obtained from all patients. The patients were fasting at least $6 \mathrm{~h}$ before fiberoptic bronchoscopy and BAE techniques.

\section{Fiberoptic bronchoscopy technique}

The fiberoptic bronchoscopy (Pentax Europe GmbH, Japan) was performed to determine the origin and cause of hemoptysis as follows:

Continuous monitoring of vital signs and oxygen saturation was performed using pulse oximetry with application of supplemental oxygen. Midazolam was administered for conscious sedation $2.5 \mathrm{mg}$ through the intravenous route. Local anesthesia of the upper airways was administered using Xylocaine 10\% spray. The bronchoscope was introduced through the nasal route; Xylocaine $2 \%$ was used to anesthetize the vocal cords, trachea, and the lower airways. The tip of the bronchoscope was advanced to visualize the bronchial tree to locate the origin of bleeding. The source of bleeding was lavaged using cold saline, which results in hemostasis, in addition to the use of topical epinephrine (1 : 20 000), which can be injected directly through the bronchoscope to stop bleeding.

Infusion of $20 \mathrm{ml}$ of saline was performed within the bronchoscopy channel and then gentle suction to collect lavage with an infusion of a total of $100-120 \mathrm{ml}$ saline to obtain BAL $40-60 \mathrm{ml}$ (equals 40-70\%); the samples were sent for micro bacteriological examination. Biopsies were taken from visible endobronchial tumors if present; also, biopsies were taken when malignancy was suspected and specimens were sent for histopathology.

\section{Technique of bronchial artery embolization}

The procedure was performed under local anesthesia; the right common femoral artery access was secured with a $5 \mathrm{Fr}, 35 \mathrm{~cm}$ sheath. A pigtail catheter was used to perform flush aortography, which enables rapid delineation of bronchial arteries and nonbronchial systemic pathologic arteries that may supply the lung lesion (e.g. subclavian arteries). Then, the culprit bronchial artery was catheterized using a 5 Fr cobra 1 (C1) catheter (Merit Medical Systems Inc., South Jordan, Utah, USA). A microcatheter [Renegade Hi-Flo, Direxion (Boston Scientific, Cork, Ireland), Repar-18 (EV3, Irvine, California, USA), or Maestro (Merit Medical Systems Inc.)] is then used for subselective catheterization of the diseased artery. The pathological branches and pseudoaneurysm were embolized with microparticles (Contour, a polyvenyl alcohol particles; Boston Scientific,). The size of the particles ranged from $150-250$ to $250-355 \mu$ ). Finally, access was closed with a clip-mediated (starclose SE) or a suture-mediated (ProGlide) closure device (Abbott Vascular, Santa Clara, USA). 


\section{Results}

There were a total of 32 patients; 20 (62.5\%) were males and $12(37.5 \%)$ were females. The age range of the patients studied was 17-73 years; 18 (56.25\%) patients were smokers. Fifteen patients underwent $\mathrm{BAE}$ as an urgent procedure for control of massive hemoptysis and 17 patients underwent $\mathrm{BAE}$ as an elective procedure.

Table 1 shows the causes of massive hemoptysis in the patients studied. The presentations of CT pulmonary angiography were in the form of aneurysmal dilation in six $(18.75 \%)$ patients, tortuosity in eight (25\%) patients, bronchopulmonary shunting in seven (21.87\%) patients, hypertrophied bronchial and nonbronchial vessels in $30(93.75 \%)$ patients, and hypervascularity in four (12.5\%) patients (Table 2 and Figs 1-3).

Fiberoptic bronchoscopy was performed to locate the origin of bleeding, which originated from the left side in $18(56.25 \%)$ cases and the right side in 14 (43.75\%) cases; biopsies were taken from visible tumors in seven (34.38\%) patients and in one patient because of mucosal thickening with hyperemia.

Table 3 shows the complications of BAE in the patients studied: fever in four (12.5\%) patients, back pain in three (9.38\%), failure in two (6.25\%), and recurrence of hemoptysis in one (3.13\%). There was no mortality.

The procedure was successful in 30 out of 32 (93.75\%) cases; however, failure of catheterization was encountered in one (3.13\%) case because of dissection

Table 1 Causes of systemic hemoptysis in the patients studied

\begin{tabular}{lc}
\hline & $N=32[n(\%)]$ \\
\hline Tuberculosis & $9(28.12)$ \\
Sarcoidosis & $4(12.5)$ \\
Malignancy & $7(21.87)$ \\
Bronchiectasis & $7(21.87)$ \\
Aspergilloma & $2(6.25)$ \\
Unknown & $3(9.37)$ \\
\hline
\end{tabular}

Table 2 Findings of computed tomography pulmonary angiography in the patients studied

\begin{tabular}{lc}
\hline & $N(\%)$ \\
\hline Aneurysmal dilation & $6(18.75)$ \\
Tortuosity & $8(25)$ \\
Bronchopulmonary shunting & $7(21.88)$ \\
Hypertrophied bronchial and nonbronchial vessels & $30(93.75)$ \\
Hypervascularity & $4(12.5)$ \\
\hline
\end{tabular}

during wire manipulation. Therefore, it was not catheterized. The second (3.13\%) case showed extensive bronchial-pulmonary shunt with severe lung destruction; thus, the procedure was deemed futile (Fig. 3). After the procedure, immediate control of hemoptysis was reported in $29(90.62 \%)$ patients.

\section{Discussion}

$\mathrm{BAE}$ is considered a substitute to surgical resection in the urgent management of massive hemoptysis. BAE can result in immediate control of hemoptysis [13].

Shigemura et al. [14] reported that TB was the most common cause of massive hemoptysis in 62 Chinese patients over the period from 2000 to 2005; this is in agreement with another study [15], which found that TB accounts for $85 \%$ of cases of massive hemoptysis. In this study, post-TB sequelae were responsible for $28.12 \%$ of cases of massive hemoptysis.

In agreement with our results, Soares et al. [16] found that TBs and bronchiectasis were the major causes of massive hemoptysis, whereas lung cancer and bronchiectasis were the main causes in the study of Fruchter et al. [17], who stated that the most common causes of massive hemoptysis that necessitate $\mathrm{BAE}$ were lung cancer and bronchiectasis. However, Soares Pires et al. [16] reported that lung cancer was the third common reason for hemoptysis. Dabo et al. [18] found that lung cancer was present only in $2.5 \%$ of patients who underwent $\mathrm{BAE}$ for control of hemoptysis.

Tom et al. [19] reported that sarcoidosis and cystic fibrosis were the main indications for $\mathrm{BAE}$, accounting for $36 \%$ out of 69 patients, whereas TB and its sequelae were present only in $8 \%$ of the patients.

Some studies have reported variable incidences of aspergilloma who underwent $\mathrm{BAE}$ for control of amount of hemopysis $[20,21]$.

Previous studies found that malignancies are present in a small number of patients compared with TB and its sequelae, with an incidence of $1-12.9 \%[22,23]$.

Most cases of massive hemoptysis arise from bronchial circulation; however, it may originate from pulmonary circulation in some pathologic conditions such as active TB [3], lung abscess [3], mycetoma, cavitating lung cancer [3], and vasculitis.

Fiberoptic bronchoscopy is useful in localization of the origin of hemoptysis in $73-93 \%$ of patients $[12,24]$, 
Figure 1
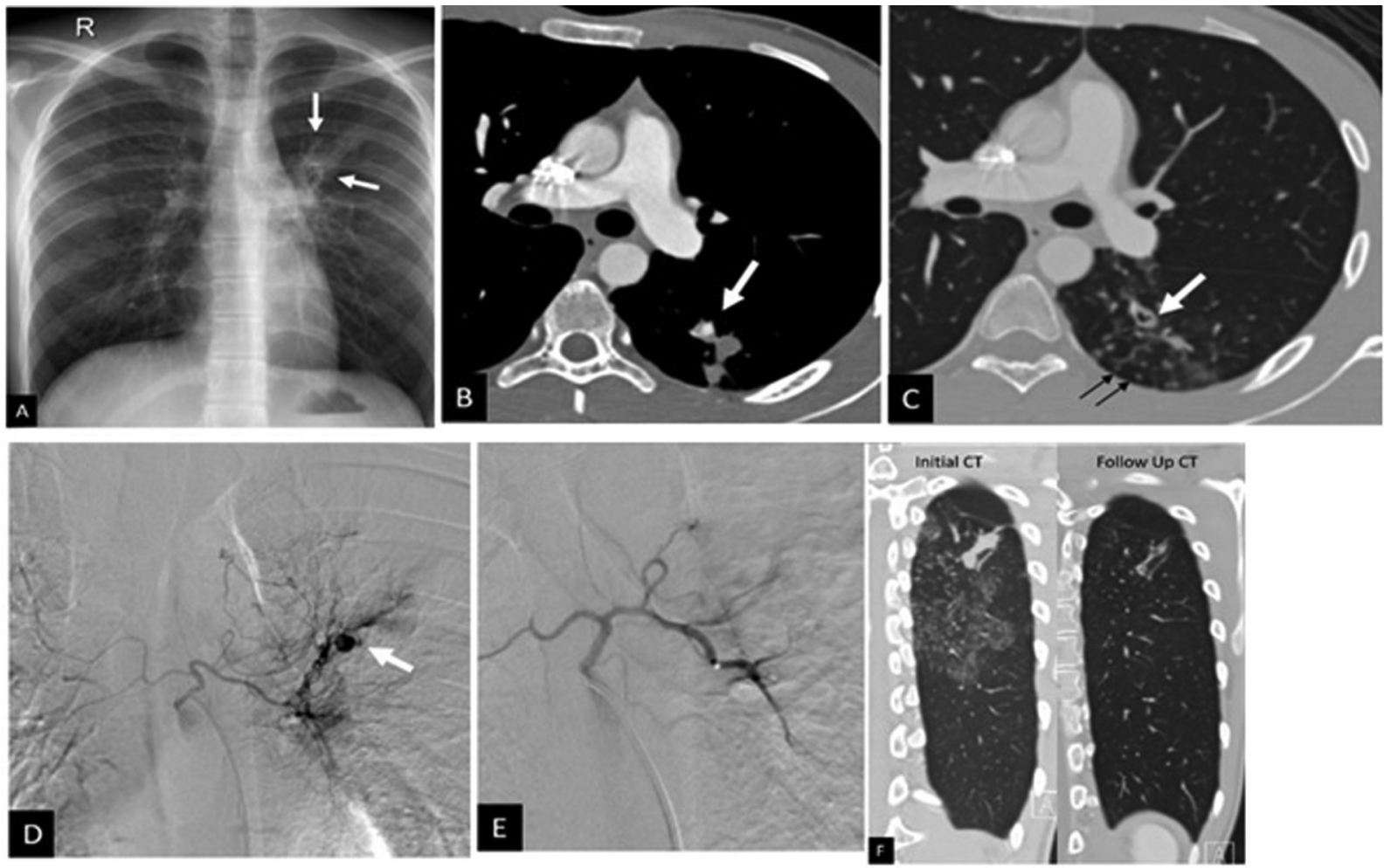

A 17-year-old man with hemoptysis. (a) Plain radiograph shows left para-hilar reticulonodular density and ill-defined opacity. (b) CTA in the mediastinal window shows a small aneurysm (arrow) in the apical segment of the left lower lobe in addition to a cavitary lesion (arrow) and tree in bud nodules (small black arrows) in (c). (d) selective bronchial angiography shows the common origin of the right and left bronchial arteries, attenuated right artery, abnormal left bronchial branches, and a small pseudoaneurysm (arrow). (e) Cessation of flow to the pathological arteries after embolization with 150-250 $\mu$ polyvenyl alcohol particles. (f) follow-up computed tomography in 3 months shows marked improvement and disappearance of the aneurysm. Patient did not have another attack since embolization. CTA, CT pulmonary angiography.

Figure 2
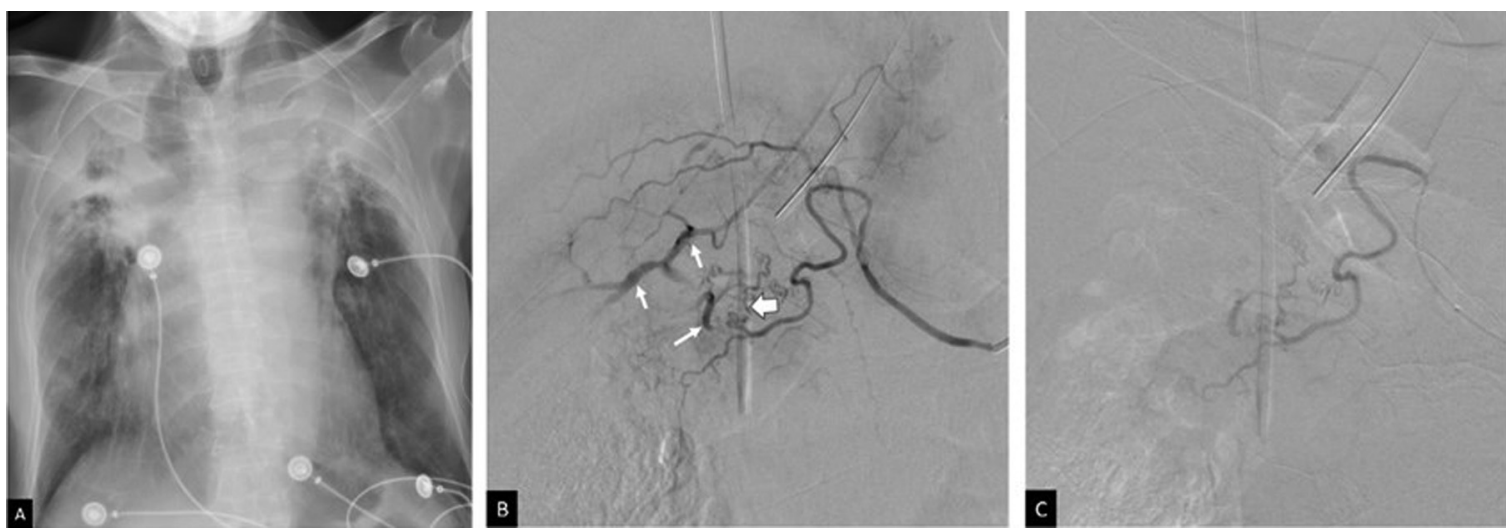

A 60-year-old man with hemoptysis. (a) Chest radiograph shows bilateral apical destructive lung changes with pleural thickening and traction on the trachea. Endoscopy confirmed bleeding from the right side. (b) Right ICBT catheterization with a $5 \mathrm{Fr}$ cobra 1 catheter shows pathological vessels (block arrow) in the upper lobe with a bronchial-pulmonary shunt (arrows). (c) Postembolization with 150-250 $\mu$ polyvenyl alcohol particles using a microcatheter. ICBT, intercostal-bronchial artery trunk.

like this study as fiberoptic bronchoscopy (FOB) determined the origin hemoptysis in some cases that were not determined by imaging neither by chest $\mathrm{X}$ ray nor CT pulmonary angiography. Remy et al. [25] performed BAE for control of hemoptysis for the first time in 1973. Multiple studies reported the success of BAE in immediate control of hemoptysis in $57-100 \%$ of patients $[22,26,27]$.

Some studies postulated that some causes of hemoptysis such as TB, lung cancer, bronchiectasis, and aspergilloma are related to an increased risk of 
Figure 3
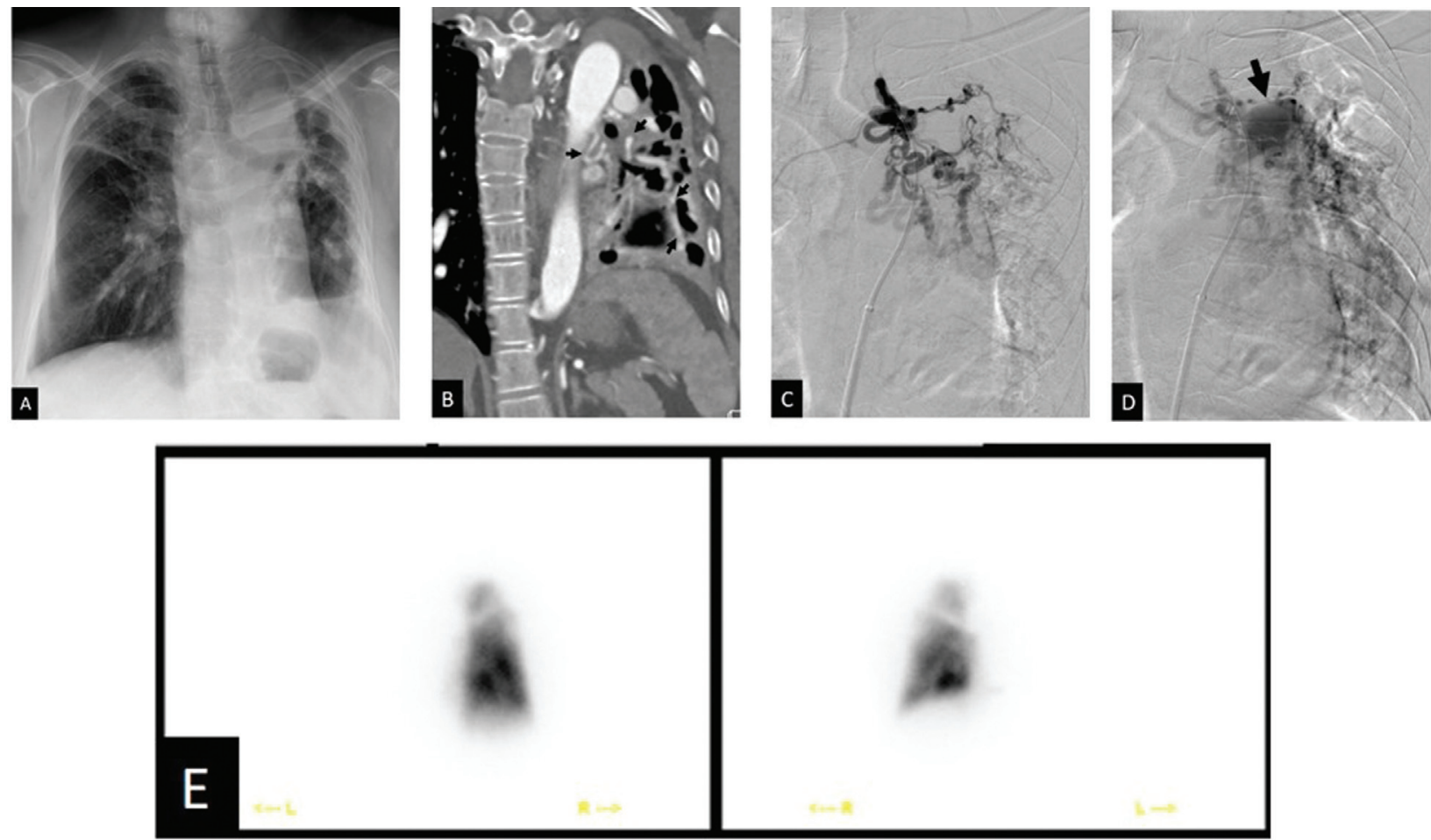

A 70-year-old woman presented with recurrent hemoptysis; bronchoscopy showed blood originating from the left side. Plain radiograph (a) shows marked destruction of left lung, bronchiectasis, volume loss, and air-fluid level. (b) CTA: marked hypertrophied and tortuous left bronchial artery (arrows). (c) Selective left bronchial angiography confirmed CTA findings; a vial of 100-250 $\mu$ was injected through a microcatheter. (d) Following particle embolization, a large shunt with the left pulmonary artery was noted (arrow). On the basis of the above findings, the benefits of embolization were questionable, and surgery was considered a safer option. (e) Preoperative assessment with ${ }^{99 m}$ Tc MAA lung perfusion confirmed the complete absence of function in the left lung. CTA, CT pulmonary angiography; MAA, macro-aggregated albumin scintigraphy.

Table 3 Complications bronchial artery embolization in the patients studied

\begin{tabular}{lc}
\hline & $N(\%)$ \\
\hline Fever & $4(12.5)$ \\
Back pain & $3(9.38)$ \\
Failure & $2(6.25)$ \\
Recurrence of hemoptysis & $1(3.13)$ \\
Mortality & 0 \\
\hline
\end{tabular}

recurrence of hemoptysis $[17,28]$. Recent studies have reported a high incidence of recurrence of hemoptysis of $30-39.2 \%$ [18,19].

In this study, no mortality was reported; in contrast to this finding, other studies [15] have reported mortality rates of $9-38 \%$ resulting from massive hemoptysis. The highest of 38\% was recorded in a series that enrolled a large number of patients with advanced carcinomas. Multiple studies have concluded that BAE is a safe and effective technique in control of hemoptysis $[17,18]$.

In this study, immediate control of hemoptysis was achieved in 90.62\%; this is in agreement of with Cremaschi et al. [29], who studied 209 patients who underwent $\mathrm{BAE}$ for hemoptysis control, and reported immediate improvement in hemoptysis in 205 (98\%) patients.

The complications that may occur with embolization are minor such as fever, chest pain, and dysphagia, which resolve in a few days [30]; this is in agreement with this study. Spinal cord infarction and/or ischemia are the most serious complications of BAE, with variable incidences 1.4-6.5\% [29].

The limitation of this study was inclusion of small number of patients with massive hemoptysis indicted for BAE according to inclusion criteria.

\section{Conclusion}

BAE can be used safely and effectively for control of massive hemoptysis; it can be used as an alternative nonsurgical option along with medical treatment or as a bridge to stabilize the patient until definitive surgical management can be performed. BAE can be considered an alternative to surgery if the patient is not fit for surgical intervention.

\section{Financial support and sponsorship}

Nil. 


\section{Conflicts of interest}

There are no conflicts of interest.

\section{References}

1 Flume PA, Yankaskas JR, Ebeling M, Hulsey T, Clark LL. Massive hemoptysis in cystic fibrosis. Chest 2005; 128:729-738.

2 Ibrahim WH. Massive haemoptysis: the definition should be revised. Eur Respir J 2008; 32:1131.

3 Khalil A, Parrot A, Nedelcu C, Fartoukh M, Marsault C, Carette MF, et al. Severe hemoptysis of pulmonary arterial origin: signs and role of multidetector row CT. Chest 2008; 133:212-219.

4 Sakr L, Dutau H. Massive hemoptysis: an update on the role of bronchoscopy in diagnosis and management. Respiration 2010; 80:38-58.

5 Zhang Y, Chen C, Jiang GN. Surgery of massive hemoptysis in pulmonary tuberculosis: immediate and long-term outcomes. J Thorac Cardiovasc Surg 2014; 148:651-656.

6 Seedat UF, Seedat F. Post-primary pulmonary TB haemoptysis - When there is more than meets the eye. Respir Med Case Rep 2018; 25:96-99.

7 Radchenko C, Alraiyes AH, Shojaee S. A systematic approach to the management of massive hemoptysis. J Thorac Dis 2017; 9:S1069-S1086.

8 Najarian KE, Morris CS. Arterial embolization in the chest. $J$ Thorac Imaging 1998; 13:93-104.

9 Marshall TJ, Jackson JE. Vascular intervention in the thorax: bronchial artery embolization for haemoptysis. Eur Radiol 1998; 7:1221-1227.

10 Khalil A, Soussan M, Mangiapan G, Fartoukh M, Parrot A, Carette MF. Utility of high-resolution chest CT scan in the emergency management of haemoptysis in the intensive care unit: severity, localization and aetiology. Br J Radiol 2007; 80:21-25.

11 McGuinness G, Beacher JR, Harkin TJ, Garay SM, Rom WM, Naidich DP. Hemoptysis: prospective high-resolution CT/bronchoscopic correlation. Chest 1994; 105:1155-1162.

12 Revel MP, Fournier LS, Hennebicque AS, Cuenod CA, Meyer G, Reynaud $\mathrm{P}$, et al. Can CT replace bronchoscopy in the detection of the site and cause of bleeding in patients with large or massive hemoptysis. Am J Roentgenol 2002; 179:1217-1224.

13 Ramakantan R, Bandekar VG, Gandhi MS, Aulakh BG, Deshmukh HL. Massive hemoptysis due to pulmonary tuberculosis: control with bronchial artery embolization. Radiology 1996; 200:691-694.

14 Shigemura N, Wan IY, Yu SC, Wong RH, Hsin MK, Thung HK, et al. Multidisciplinary management of life-threatening massive hemoptysis: a 10-year experience. Ann Thorac Surg 2009; 87:849-853.

15 Knott-Craig CJ, Oostuizen G, Rossouw G, Joubert JR, Barnard PM Management and prognosis of massive hemoptysis: recent experience with 120 patients. J Thorac Cardiovasc Surg 1993; 105:394-397.

16 Soares Pires F, Teixeira N, Coelho F, Damas C. Hemoptysis - etiology, evaluation and treatment in a university hospital. Rev Port Pneumol 2011; 17:7-14
17 Fruchter O, Schneer S, Rusanov V, Belenky A, Kramer MR. Bronchial artery embolization for massive hemoptysis: long-term follow-up. Asian Cardiovasc Thorac Ann 2015; 23:55-60.

18 Dabo H, Gomes R, Marinho A, Madureira M, Paquete J, Morgado P. Bronchial artery embolisation in management of hemoptysis - a retrospective analysis in a tertiary university hospital. Rev Port Pneumol (2006) 2016; 22:34-38.

19 Tom LM, Palevsky HI, Holsclaw DS, Trerotola SO, Dagli M, Mondschein J. Recurrent bleeding, survival, and longitudinal pulmonary function following bronchial artery embolization for hemoptysis in a U.S. adult population. $J$ Vasc Interv Radiol 2015; 26:1806-1813.

20 van den Heuvel MM, Els Z, Koegelenberg CF, Naidu KM, Bolliger CT, Diacon $\mathrm{AH}$. Risk factors for recurrence of haemoptysis following bronchia artery embolisation for life-threatening haemoptysis. Int J Tuberc Lung Dis 2007; 11:909-914

21 Yoo DH, Yoon CJ, Kang SG, Burke CT, Lee JH, Lee CT. Bronchial and nonbronchial systemic artery embolization in patients with major hemoptysis: safety and efficacy of N-butyl cyanoacrylate. $A m J$ Roentgenol 2011; 196:W199-W204.

22 Swanson KL, Johnson M, Prakash UBS, McKusick MA, Andrews JC, Stanson AW. Bronchial artery embolization, experience with 54 patients. Chest 2002; 121:789-795.

23 Bhalla A, Kandasamy D, Veedu P, Mohan A, Gamanagatti S. A retrospective analysis of 334 cases of hemoptysis treated by bronchia artery embolization. Oman Med J 2015; 30:119-128.

24 Hsiao El, Kirsch CM, Kagawa FT, Wehner JH, Jensen WA, Baxter RB. Utility of fiberoptic bronchoscopy before bronchial artery embolization for massive hemoptysis. Am J Roentgenol 2001 177:861-867.

25 Remy J, Voisin C, Ribet M, Dupuis C, Beguery P, Tonnel AB, et al. Treatment, by embolization, of severe or repeated hemoptysis associated with systemic hypervascularization. Nouv Presse Med 1973 2:2060-2068.

26 Barben J, Robertson D, Olinsky A, Ditchfield M. Bronchial artery embolization for hemoptysis in young patients with cystic fibrosis. Radiology 2002; 224:124-130.

27 Fartoukh M, Khalil A, Louis L, Carette MF, Bazelly B, Cadranel J, et al. An integrated approach to diagnosis and management of severe haemoptysis in patients admitted to the intensive care unit: a case series from a referra centre. Respir Res 2007; 8:11.

28 Garcia-Olive I, Sanz-Santos J, Centeno C, Andreo F, Munoz-Ferrer A Serra $\mathrm{P}$, et al. Results of bronchial artery embolization for the treatment of hemoptysis caused by neoplasm. J Vasc Interv Radiol 2014 25:221-228.

29 Cremaschi $\mathrm{P}$, Nascimbene C, Vitulo P, Catanese C, Rota L, Barazzoni GC, et al. Therapeutic embolization of bronchial artery: a successful treatment in 209 cases of relapse hemoptysis, '. Angiology 1993; 44:295-299.

30 Pollak J. Radiologic management of massive hemoptysis. Contemp Intern Med 1998; 10:28-35. 